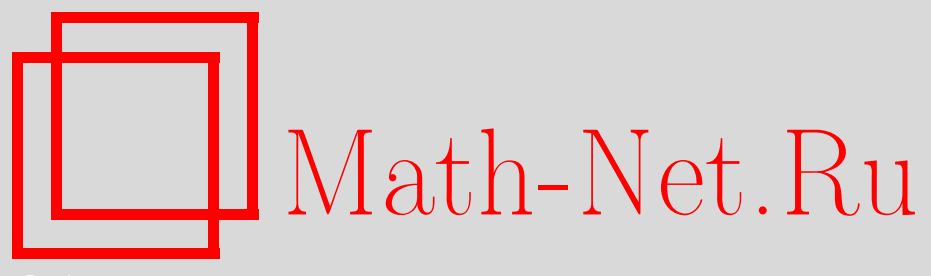

М. Е. Палистрант, В. А. Урсу, Выход за рамки теоремы Мигдала в теории двухзонной сверхпроводимости, TMФ, 2006, том 149, номер 1, 111-126

DOI: https://doi.org/10.4213/tmf3835

Использование Общероссийского математического портала Math-Net.Ru подразумевает, что вы прочитали и согласны с пользовательским соглашением http://www . mathnet.ru/rus/agreement

Параметры загрузки:

IP : 54.92 .164 .108

26 апреля 2023 г., 14:53:14

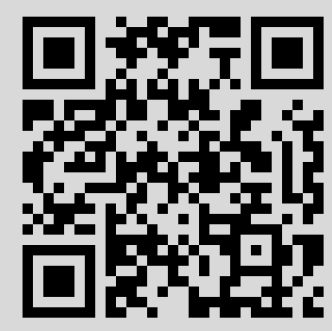




\title{
ВЫХОД ЗА РАМКИ ТЕОРЕМЫ МИГДАЛА В ТЕОРИИ ДВУХЗОННОЙ СВЕРХПРОВОДИМОСТИ
}

\begin{abstract}
Предложена теория сверхпроводимости двухзонных неадиабатических систем с сильными электронными корреляциями в линейном приближении по неадиабатичности. В предположении слабого электрон-фононного взаимодействия получены аналитические выражения для вершинной и "пересекающейся" функций для каждой из двух зон. С учетом диаграмм с пересечением двух линий электрон-фононного взаимодействия (выход за рамки теоремы Мигдала) определены массовые операторы функций Грина и на их основе получены основные уравнения теории сверхпроводимости двухзонных систем. Найдено аналитическое выражение для температуры сверхпроводящего перехода $T_{\mathrm{c}}$, которое отличается от случая обычных двухзонных систем существенной перенормировкой входящих в него величин, связанной с эффектами неадиабатичности и сильными электронными корреляциями. Изучена зависимость величины $T_{\mathrm{c}}$ и изотопического коэффициента $\alpha$ от параметра Мигдала $m=\omega_{0} / \varepsilon_{\mathrm{F}}$. Показано, что учет перекрытия энергетических полос на поверхности Ферми и эффектов неадиабатичности при малых значениях передаваемого импульса $\left(q \ll 2 p_{\text {F }}\right.$ )

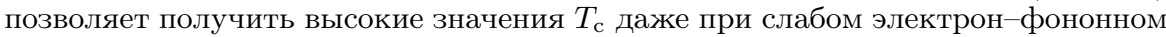
взаимодействии.
\end{abstract}

Ключевые слова: сверхпроводимость, неадиабатическая система, электрон-фононное взаимодействие, теорема Мигдала.

\section{1. ВВЕДЕНИЕ}

Прошло около 20 лет со дня открытия высокотемпературных сверхпроводящих материалов [1]. Все это время велись интенсивные экспериментальные и теоретические исследования свойств этих материалов в нормальной и сверхпроводящей фазах. Установлено существенное отличие физических свойств этих материалов от свойств обычных сверхпроводников. Новые материалы обладают рядом особенностей: сильной анизотропией кристаллических свойств (слоистой структурой), сильными электронными корреляциями, сильным электрон-фононным взаимодействием, наличием точек высокой симметрии в импульсном пространстве, перекрытием энергетических полос на поверхности Ферми, малыми значениями энергии Ферми и др. Сложность данных систем затрудняет решение одной из основных проблем

*Academy of Sciences of Moldova, Institute of Applied Physics, Chisinau, Moldova. E-mail: mepalistrant@yandex.ru, ursu@hotbox.ru 
современной физики - выявление механизма высокотемпературной сверхпроводимости. Одним из возможных вариантов решения этой проблемы является последовательное рассмотрение присущих этим материалам особенностей и изучение их влияния на термодинамические и магнитные свойства.

Так, например, для изучения влияния на сверхпроводимость перекрытия энергетических полос на поверхности Ферми, приводящего к возникновению двух энергетических щелей, была использована модель Москаленко [2] (см. также работу [3]). На основании этой модели задолго до открытия высокотемпературной сверхпроводимости была построена теория сверхпроводимости для обычных сверхпроводников (чистых и примесных), историю развития этой теории можно найти в [4].

Попытка применить эту теорию к материалам с высокотемпературной сверхпроводимостью была предпринята, например, в работе [5]. Как следует из рассуждений работы [5], в рамках этой теории можно описать довольно большое количество экспериментальных данных, связанных со сверхпроводящими свойствами металооксидных соединений.

С другой стороны, возникает ряд теоретических вопросов, в частности, следует объяснить роль неадиабатичности и сильных электронных корреляций в высокотемпературных материалах. Речь идет о малых значениях энергии Ферми $\left(\varepsilon_{\mathrm{F}} \ll \omega_{0}\right)$ или ее соизмеримости с энергией Дебая $\left(\varepsilon_{\mathrm{F}} \sim \omega_{0}\right)$ и о больших значениях кулоновского взаимодействия электронов В рамках теории Боголюбова-Элиашберга-Мигдала эти факторы не могут способствовать возникновению высокотемпературной сверхпроводимости. Более того, эта теория не применима для описания неадиабатических систем, в которых нарушается теорема Мигдала [6]. Возникает необходимость рассмотрения вершинных и "пересекающихся" диаграмм по электрон-фононному взаимодействию, что помогает учесть дополнительные многочастичные эффекты при определении функций Грина.

Сверхпроводимости в неадиабатических системах и построению теории, выходящей за рамки теоремы Мигдала, посвящено много работ (см. обзор [7]). Из этих исследований вытекает, что вершинные функции при $\varepsilon_{\mathrm{F}} \sim \omega_{0}$ оказываются отрицательными и, следовательно, уменьшают константу электрон-фононного взаимодействия, не приводя к высоким значениям температуры сверхпроводящего перехода $T_{\mathrm{c}}$. Противоположную картину мы имеем в тех случаях, когда передаваемый импульс при электрон-фононном взаимодействии мал $\left(q \ll 2 p_{\mathrm{F}}\right)$. Такая ситуация возможна, в частности, при наличии в системе сильных электронных корреляций [8]. В работах [9], [10] рассмотрена однозонная неадиабатическая система с серединным заполнением энергетической зоны и выполнено обрезание электрон-фононного взаимодействия по малому значению передаваемого импульса $q_{\mathrm{c}} \ll 2 p_{\mathrm{F}}$. При таком подходе вершинные функции $P_{\mathrm{V}}$ и “пересекающиеся" функции $P_{\mathrm{C}}$ оказываются положительными и способны существенно увеличить константу электрон-фононного взаимодействия, в результате чего значение температуры сверхпроводящего перехода доходит до величин, присущих материалам с высокотемпературной сверхпроводимостью. Положительные значения функций $P_{\mathrm{V}}$ и $P_{\mathrm{C}}$ возможны также в неадиабатических системах с особенностями Ван Хова в электронном энергетическом спектре. В частности, это хорошо видно на примере "протяженной" особенности в электронном энергетическом спектре [11], обнаруженной экспериментально в итриевой керамике [12]. 
Отметим, что исследования термодинамических свойств однозонных сверхпроводящих неадиабатических систем с переменной плотностью носителей заряда (чистых и с магнитной примесью) содержатся в серии работ одного из авторов данной статьи [13], [14]. Эти исследования показывают, что неадиабатичность вместе с сильными электронными корреляциями благоприятно влияет на сверхпроводящее состояние однозонных систем.

Последние пять лет очень интенсивно ведутся поиски новых сверхпроводящих материалов. Особый интерес вызывает соединение $\mathrm{Mg} \mathrm{B}_{2}$, имеющее температуру сверхпроводящего перехода $T_{\mathrm{c}} \sim 40 \mathrm{~K}[15]$. Это вещество обладает рядом аномалий физических свойств (см., например, обзоры [16], [17]) и, как выяснилось, эти аномалии связаны с анизотропными свойствами системы и, в частности, с наличием перекрытия энергетических полос на поверхности Ферми. В основе описания свойств соединения $\mathrm{Mg} \mathrm{B}_{2}$ лежит теория сверхпроводимости для систем с перекрывающимися на поверхности Ферми энергетическими зонами [2], [16]-[18]. Наряду с этим при определенных условиях в соединении $\mathrm{Mg} \mathrm{B}_{2}$ могут проявляться эффекты недиабатичности, поскольку для этого соединения параметр Мигдала $m=\omega_{0} / \varepsilon_{\mathrm{F}} \approx 0,1$ [19].

Таким образом, современные высокотемпературные сверхпроводники (оксидные металлы, фулерены, органические системы, борид магния) являются неадиабатическими системами. Кроме того, электронный энергетический спектр этих систем содержит особенность: перекрытие энергетических полос на поверхности Ферми, что подтверждается зонными расчетами (см., например, [20]). Указанные выше обстоятельства являются серьезным стимулом для построения теории сверхпроводимости в двухзонных неадиабатических системах Этому посвящена данная работа.

В разделе 2 на основании гамильтониана типа гамильтониана Фрелиха получены уравнения для температурных электронных функций Грина и выражения для массовых операторов $M$ и $\Sigma$, а также выполнен переход к зонному $n \vec{k} \Omega$-представлению. В разделе 3 даны определения вершинных функции $P_{\mathrm{V} n}$ и "пересекающихся" функций $P_{\mathrm{C} n}$ для $n$-й зоны, $n=1,2$, а также получена система уравнений для параметров порядка двухзонной системы. В разделе 4 приведено уравнение для температуры сверхпроводящего перехода $T_{\mathrm{c}}$ и найдено его решение. Определено также значение коэффициента изотопического эффекта $\alpha$. В разделе 5 выполнены численные расчеты и проведен анализ результатов.

\section{2. ГАМИЛЬТОНИАН СИСТЕМЫ И ФУНКЦИИ ГРИНА}

Рассмотрим гамильтониан типа гамильтониана Фрелиха:

$$
H=H_{0}+H_{1}
$$

где $H_{0}$ - гамильтониан невзаимодействующих электронов и фононов, а $H_{1}$ - гамиль-

5 Теоретическая и математическая физика, т. 149, № 1, 2006 г. 
тониан электрон-фононного взаимодействия, определяемый выражением

$$
H_{1}=\sum_{\sigma} \int d \vec{x} \psi_{\sigma}^{+}(\vec{x}) \psi_{\sigma}(\vec{x}) \varphi(\vec{x}) .
$$

Здесь $\psi_{\sigma}^{+}(\vec{x})$ и $\psi_{\sigma}(\vec{x})$ - сответственно операторы рождения и уничтожения электрона со спином $\sigma$ в точке $\vec{x}, \varphi(\vec{x})$ - фононное поле. На основании гамильтониана (1) и диаграммного метода теории возмущений [21] можно получить систему уравнений для нормальной $G\left(x, x^{\prime}\right)$ и аномальной $\widetilde{F}\left(x, x^{\prime}\right)$ функций Грина:

$$
\begin{gathered}
G\left(x, x^{\prime}\right)=G^{0}\left(x, x^{\prime}\right)+\iint d x_{1} d x_{2} G^{0}\left(x, x_{1}\right) M\left(x_{1}, x_{2}\right) G\left(x_{2}, x^{\prime}\right)- \\
\quad-\iint d x_{1} d x_{2} G^{0}\left(x, x_{1}\right) \Sigma\left(x_{1}, x_{2}\right) \widetilde{F}\left(x_{2}, x^{\prime}\right), \\
\widetilde{F}\left(x, x^{\prime}\right)=\int d x_{1} d x_{2} G_{0}\left(x_{1}, x\right) \widetilde{\Sigma}\left(x_{1}, x_{2}\right) G\left(x_{2}, x^{\prime}\right)+ \\
+\int d x_{1} d x_{2} G^{0}\left(x_{1}, x\right) M\left(x_{2}, x_{1}\right) \widetilde{F}\left(x_{2}, x^{\prime}\right),
\end{gathered}
$$

где $G^{0}\left(x, x^{\prime}\right)$ - функция Грина свободных электронов, $G\left(x, x^{\prime}\right)$ и $F\left(x, x^{\prime}\right)$ - полные электронные функции Грина сверхпроводящего состояния, $\Sigma\left(x_{1}, x_{2}\right)$ и $M\left(x_{1}, x_{2}\right)-$ массовые операторы (недиагональный и диагональный, соответственно), знак ${ }_{\text {над }}$ функцией обозначает комплексное сопряжение.

Поскольку в (1) отсутствуют спиновые взаимодействия, при получении системы (3) были использованы соотношения

$$
G_{\alpha \beta}\left(x, x^{\prime}\right)=\delta_{\alpha, \beta} G\left(x, x^{\prime}\right), \quad \widetilde{F}_{\alpha \alpha^{\prime}}\left(x, x^{\prime}\right)=-g_{\alpha, \alpha^{\prime}} \widetilde{F}\left(x, x^{\prime}\right),
$$

где

$$
g_{\alpha, \alpha^{\prime}}=\delta_{\alpha,-\alpha^{\prime}}\left(\delta_{\alpha \uparrow}-\delta_{\alpha \downarrow}\right)=-g_{\alpha^{\prime}, \alpha},
$$

$\alpha, \alpha^{\prime}$ - спиновые индексы, принимающие два значения: $\uparrow$ (вверх) и $\downarrow$ (вниз). Массовые операторы (диагональный $M$ и недиагональный $\Sigma$ ) при температуре, близкой к критической $\left(T \sim T_{\mathrm{c}}\right)$, имеют вид

$$
\begin{aligned}
M\left(x_{1}, x_{2}\right)=- & D\left(x_{1}, x_{2}\right) G\left(x_{1}, x_{2}\right)+\iint d x_{3} d x_{4} D\left(x_{4}, x_{2}\right) D\left(x_{1}, x_{3}\right) \times \\
& \times G\left(x_{1}, x_{4}\right) G\left(x_{4}, x_{3}\right) G\left(x_{3}, x_{2}\right), \\
\Sigma\left(x_{1}, x_{2}\right)=- & D\left(x_{1}, x_{2}\right) F\left(x_{1}, x_{2}\right)+\iint d x_{3} d x_{4} D\left(x_{1}, x_{3}\right) D\left(x_{4}, x_{2}\right) \times \\
& \times\left[G\left(x_{1}, x_{4}\right) G\left(x_{2}, x_{3}\right) F\left(x_{4}, x_{3}\right)+G\left(x_{3}, x_{4}\right) F\left(x_{1}, x_{4}\right) G\left(x_{2}, x_{3}\right)+\right. \\
+ & \left.F\left(x_{3}, x_{2}\right) G\left(x_{1}, x_{4}\right) G\left(x_{4}, x_{3}\right)-F\left(x_{1}, x_{3}\right) \widetilde{F}\left(x_{3}, x_{4}\right) F\left(x_{4}, x_{2}\right)\right],
\end{aligned}
$$

где

$$
D\left(x, x^{\prime}\right)=\frac{1}{\beta} \sum_{\vec{q}, \omega} D(\vec{q}, \omega) e^{-i \vec{q}\left(v e c x-\vec{x}^{\prime}\right)-i \omega\left(\tau-\tau^{\prime}\right)}
$$

- функция Грина фононов. Из этих формул следует, что наряду с диаграммами, которые учитываются в массовых операторах адиабатических систем (первые слагаемые в (6), (7)), мы вводим в рассмотрение также члены с пересечением двух линий 
электрон-фононного взаимодействия. Это соответствует учету вершинных поправок и отвечает выходу теории сверхпроводимости за рамки теоремы Мигдала [6]. Перейдем в $(3),(6)$ и $(7)$ к $n \vec{k} \Omega$-представлению в соответствии с соотношениями для функций Грина:

$$
\begin{aligned}
& G\left(x, x^{\prime}\right)=\frac{1}{\beta} \sum_{\vec{k} \vec{k}^{\prime}} \sum_{n m} \sum_{\Omega} G_{n m}\left(\vec{k}, \vec{k}^{\prime}, \Omega\right) \psi_{n \vec{k}}(\vec{x}) \psi_{m \vec{k}^{\prime}}^{*}\left(\vec{x}^{\prime}\right) e^{-i \Omega\left(\tau-\tau^{\prime}\right)}, \\
& F\left(x, x^{\prime}\right)=\frac{1}{\beta} \sum_{\vec{k} \vec{k}^{\prime}} \sum_{n m} \sum_{\Omega} F_{n m}\left(\vec{k}, \vec{k}^{\prime}, \Omega\right) \psi_{n \vec{k}}(\vec{x}) \psi_{m \vec{k}^{\prime}}\left(\vec{x}^{\prime}\right) e^{-i \Omega\left(\tau-\tau^{\prime}\right)}, \\
& \widetilde{F}\left(x, x^{\prime}\right)=\frac{1}{\beta} \sum_{\vec{k} \vec{k}^{\prime}} \sum_{n m} \sum_{\Omega} \widetilde{F}_{n m}\left(\vec{k}, \vec{k}^{\prime}, \Omega\right) \psi_{n \vec{k}}^{*}(\vec{x}) \psi_{m \vec{k}^{\prime}}^{*}\left(\vec{x}^{\prime}\right) e^{-i \Omega\left(\tau-\tau^{\prime}\right)},
\end{aligned}
$$

а также для массовых операторов

$$
\begin{aligned}
& M\left(x, x^{\prime}\right)=\frac{1}{\beta} \sum_{\vec{k} \vec{k}^{\prime}} \sum_{n m} \sum_{\Omega} M_{n m}\left(\vec{k}, \vec{k}^{\prime}, \Omega\right) \psi_{n \vec{k}}(\vec{x}) \psi_{m \vec{k}^{\prime}}^{*}\left(\vec{x}^{\prime}\right) e^{-i \Omega\left(\tau-\tau^{\prime}\right)}, \\
& \Sigma\left(x, x^{\prime}\right)=\frac{1}{\beta} \sum_{\vec{k} \vec{k}^{\prime}} \sum_{n m} \sum_{\Omega} \Sigma_{n m}\left(\vec{k}, \vec{k}^{\prime}, \Omega\right) \psi_{n \vec{k}}(\vec{x}) \psi_{m \vec{k}^{\prime}}\left(\vec{x}^{\prime}\right) e^{-i \Omega\left(\tau-\tau^{\prime}\right)}, \\
& \widetilde{\Sigma}\left(x, x^{\prime}\right)=\frac{1}{\beta} \sum_{\vec{k} \vec{k}^{\prime}} \sum_{n m} \sum_{\Omega} \widetilde{\Sigma}_{n m}\left(\vec{k}, \vec{k}^{\prime}, \Omega\right) \psi_{n \vec{k}}^{*}(\vec{x}) \psi_{m \vec{k}^{\prime}}^{*}\left(\vec{x}^{\prime}\right) e^{-i \Omega\left(\tau-\tau^{\prime}\right)},
\end{aligned}
$$

где $\psi_{n \vec{k}}(\vec{x})=e^{i \vec{k} \vec{x}} U_{n \vec{k}}(\vec{x})-$ функция Блоха, $x=(\vec{x}, \tau), x^{\prime}=\left(\vec{x}^{\prime}, \tau^{\prime}\right), \beta=T^{-1}, \Omega=$ $(2 \nu+1) \pi T, \omega=2 \nu \pi T, \nu=0, \pm 1, \pm 2, \ldots$ - мацубаровские частоты: электронная и фононная, соответственно.

Подставив (8) и (9) в (3) и выполнив необходимые операции, получим систему уравнений для $G_{n m}\left(\vec{k}, \vec{k}^{\prime}, \Omega\right)$ и $\widetilde{F}_{n m}\left(\vec{k}, \vec{k}^{\prime}, \Omega\right)$. Решение этой системы и термодинамические свойства двухзонного сверхпроводника без учета эффектов неадиабатичности можно найти в работах [22]. Заметим, что при таком подходе учитываются всевозможные сверхпроводящие спаривания как между электронами в каждой зоне, так и между электронами различных энергетических зон.

В дальнейшем мы ограничимся диагональным по индексам энергетических зон приближением:

$$
G_{n m}\left(\vec{k}, \vec{k}^{\prime}, \Omega\right)=\delta_{\vec{k} \vec{k}^{\prime}} \delta_{n m} G_{n}(\vec{k}, \Omega), \quad \widetilde{F}_{n m}\left(\vec{k}, \vec{k}^{\prime}, \Omega\right)=\delta_{\vec{k},-\vec{k}^{\prime}} \delta_{n m} \widetilde{F}_{n}(\vec{k}, \Omega) .
$$

Если не принимать во внимание эффекты, связанные с неадиабатичностью системы, это приближение приводит к двухзонной модели Москаленко [2], которая учитывает процессы, связанные с образованием куперовских пар в каждой энергетической зоне и их туннелирование из одной зоны в другую.

В приближении (10) решение системы уравнений (3) в $n \vec{k} \Omega$-представлении можно привести к виду

$$
G_{n}(\vec{k}, \Omega)=-\frac{i Z_{n}(\Omega) \Omega+\tilde{\varepsilon}_{n}(\vec{k})}{A_{n}(\vec{k}, \Omega)}, \quad \widetilde{F}_{n}(\vec{k}, \Omega)=\frac{\widetilde{\Sigma}_{n}(k, \Omega)}{A_{n}(\vec{k}, \Omega)}, \quad F_{n}(\vec{k}, \Omega)=\frac{\Sigma_{n}(\vec{k}, \Omega)}{A_{n}(\vec{k}, \Omega)},
$$


где

$$
\begin{gathered}
A_{n}(\vec{k}, \Omega)=\Omega^{2} Z_{n}^{2}(\Omega)+\tilde{\varepsilon}_{n}^{2}(\vec{k})+\Sigma_{n}(\vec{k}, \Omega) \widetilde{\Sigma}_{n}(\vec{k}, \Omega), \\
Z_{n}(\Omega)=1-\frac{1}{\Omega} \operatorname{Im} M_{n}(\vec{k}, \Omega), \quad \tilde{\varepsilon}_{n}(\vec{k})=\varepsilon_{n}(\vec{k})+\operatorname{Re} M_{n}(\vec{k}, \Omega),
\end{gathered}
$$

$\varepsilon_{n}(\vec{k})$ - энергия электронов в $n$-й зоне, $\tilde{\varepsilon}_{m}(\vec{k})$ - ее значение, перенормированное вследствие взаимодействия электронов с фононами, $M_{n}$ и $\Sigma_{n}$ - массовые операторы, определяемые выражениями (6), (7), в $n \vec{k} \Omega$-представлении. Они учитывают диаграммы, которые нарушают теорему Мигдала.

\section{3. МАССОВЫЕ ОПЕРАТОРЫ И ВЕРШИННЫЕ ФУНКЦИИ}

Выполнив переход к $n \vec{k} \Omega$-представлению в определениях массовых операторов (6) и (7) в соответствии с разложениями (8) и (9), получаем

$$
\begin{aligned}
M_{m}(\vec{p}, \Omega)=- & \frac{1}{\beta} \sum_{\vec{p}_{1}} \sum_{\Omega_{1}} \sum_{n} D\left(\vec{p}-\vec{p}_{1}, \Omega-\Omega_{1}\right) g_{m n}^{2}\left(\vec{p}, \vec{p}_{1}\right) G_{n}\left(\vec{p}_{1}, \Omega_{1}\right)+ \\
& +\frac{1}{\beta^{2}} \sum_{\vec{p}_{1}, \vec{p}_{2}} \sum_{\Omega_{1}, \Omega_{2}} D\left(\vec{p}-\vec{p}_{1}, \Omega-\Omega_{1}\right) \times \\
& \times D\left(\vec{p}-\vec{p}_{2}, \Omega-\Omega_{2}\right) g_{m m}^{2}\left(\vec{p}, \vec{p}_{1}\right) g_{m m}^{2}\left(\vec{p}, \vec{p}_{2}\right) \times \\
& \times G_{m}\left(\vec{p}_{1}, \Omega_{1}\right) G_{m}\left(\vec{p}_{1}+\vec{p}_{2}-\vec{p}, \Omega_{1}+\Omega_{2}-\Omega\right) G_{m}\left(\vec{p}_{2}, \Omega_{2}\right), \\
\Sigma_{m}(\vec{p}, \Omega)=- & \frac{1}{\beta} \sum_{\vec{p}_{1}} \sum_{\Omega_{1}} \sum_{n_{1}} D\left(\vec{p}-\vec{p}_{1}, \Omega-\Omega_{1}\right) g_{m n_{1}}^{2}\left(\vec{p}, \vec{p}_{1}\right) F_{n_{1}}\left(\vec{p}_{1}, \Omega_{1}\right)+ \\
& +\frac{1}{\beta^{2}} \sum_{\vec{p}_{1}, \vec{p}_{2}} \sum_{\Omega_{1}, \Omega_{2}} D\left(\vec{p}-\vec{p}_{1}, \Omega-\Omega_{1}\right) \times \\
& \times D\left(\vec{p}-\vec{p}_{2}, \Omega-\Omega_{2}\right) g_{m m}^{2}\left(\vec{p}, \vec{p}_{2}\right) g_{m m}^{2}\left(\vec{p}, \vec{p}_{1}\right)\left[G_{m}\left(\vec{p}_{2}, \Omega_{2}\right) \times\right. \\
& \times F_{m}\left(\vec{p}_{1}, \Omega_{1}\right) G_{m}\left(\vec{p}_{2}+\vec{p}_{1}-\vec{p}, \Omega_{2}+\Omega_{1}-\Omega\right)+ \\
& +F_{m}\left(\vec{p}_{1}, \Omega_{1}\right) G_{m}\left(\vec{p}_{2}, \Omega_{2}\right) G_{m}\left(\vec{p}_{2}-\vec{p}_{1}-\vec{p}, \Omega_{2}-\Omega_{1}-\Omega\right)+ \\
& \left.+F_{m}\left(\vec{p}_{1}, \Omega_{1}\right) G_{m}\left(\vec{p}-\vec{p}_{1}-\vec{p}_{2}, \Omega-\Omega_{1}-\Omega_{2}\right) G_{m}\left(-\vec{p}_{2},-\Omega_{2}\right)\right] .
\end{aligned}
$$

Здесь было использовано диагональное по индексам зон приближение (10) и приближение для матричных элементов электрон-фононного взаимодействия, отвечающее образованию куперовских пар в каждой энергетической зоне и их туннелированию как целое из одной зоны в другую. Эти процессы приводят к необходимости учета членов, содержащих константы электрон-фононного взаимодействия типа

$$
g_{m n}^{2}\left(\vec{p}, \vec{p}_{1}\right)=g^{2}\left(\vec{p}, \vec{p}_{1}\right)\left|\chi\left(m \vec{p}_{\mathrm{F}}, n \vec{p}_{1 \mathrm{~F}}\right)\right|^{2}
$$

где

$$
\chi\left(m \vec{p}_{\mathrm{F}}, n \vec{p}_{1 \mathrm{~F}}\right)=\int_{V_{0}} d \vec{r} U_{m \vec{p}_{\mathrm{F}}}^{*}(\vec{r}) U_{n \vec{p}_{1 \mathrm{~F}}}(\vec{r}),
$$

$m, n=1,2, V_{0}$ - объем элементарной ячейки кристалла.

На основании выражение (13) и (14) нетрудно заметить, что вершинные поправки в массовых операторах вносят дополнительный вклад в значения констант внутризонного электрон-электронного взаимодействия $g_{m m}^{2}$ и не влияют на константы межзонного взаимодействия $g_{m n}^{2}(m \neq n)$. 
Предположим далее, что в системе присутствуют условия, приводящие при электрон-фононном взаимодействии к малым значениям передаваемого импульса $\vec{q}$. Таким условием может быть, в частности, наличие сильных электронных корреляций [8] В соответствии с этим представим $g_{m n}^{2}\left(\vec{p}, \vec{p}^{\prime}\right)$ в виде

$$
g_{m n}^{2}\left(\vec{p}, \vec{p}^{\prime}\right)=g_{m n}^{2} \eta \theta\left(q_{\mathrm{c}}-\left|\vec{p}-\vec{p}^{\prime}\right|\right),
$$

где $q_{\mathrm{c}}$ - импульс обрезания электрон-фононного взаимодействия, а $\eta$ определяется соотношением

$$
\eta\left\langle\left\langle\theta\left(q_{\mathrm{c}}-\left|\vec{p}-\vec{p}^{\prime}\right|\right)\right\rangle\right\rangle_{\mathrm{FS}}=1,
$$

т.е. $\left\langle\left\langle g_{m n}^{2}\left(\vec{p}, \vec{p}^{\prime}\right)\right\rangle\right\rangle_{\mathrm{FS}}=g_{m n}^{2}$. Здесь $\langle\langle\ldots\rangle\rangle_{\mathrm{FS}}-$ усреднение по поверхности Ферми. Для фононного пропагатора мы используем простой энштейновский спектр с частотой $\omega_{0}$,

$$
D\left(\Omega, \Omega_{1}\right)=-\frac{\omega_{0}^{2}}{\left(\Omega-\Omega_{1}\right)^{2}+\omega_{0}^{2}} .
$$

Введем вершинные и "пересекающиеся" функции $(n=1,2)$

$$
\begin{aligned}
P_{\mathrm{V} n}\left(\vec{p}, \vec{p}_{1}, \Omega, \Omega_{1}\right)=- & \frac{1}{\beta} \frac{\eta}{N_{n}} \sum_{\vec{p}_{2}} \sum_{\Omega_{2}} \theta\left(q_{\mathrm{c}}-\left|\vec{p}-\vec{p}_{2}\right|\right) D\left(\Omega, \Omega_{2}\right) G_{n}\left(\vec{p}_{2}, \Omega_{2}\right) \times \\
& \times G_{n}\left(\vec{p}_{1}+\vec{p}_{2}-\vec{p}, \Omega_{1}+\Omega_{2}-\Omega\right), \\
P_{\mathrm{C} n}\left(\vec{p}, \vec{p}_{1}, \Omega, \Omega_{1}\right)=- & \frac{1}{\beta} \frac{\eta}{N_{n}} \sum_{\vec{p}_{2}} \sum_{\Omega_{2}} \theta\left(q_{\mathrm{c}}-\left|\vec{p}-\vec{p}_{2}\right|\right) D\left(\Omega, \Omega_{2}\right) G_{n}\left(\vec{p}_{2}, \Omega_{2}\right) \times \\
& \times G_{n}\left(\vec{p}_{1}-\vec{p}_{2}-\vec{p}, \Omega_{1}-\Omega_{2}-\Omega\right) .
\end{aligned}
$$

Воспользовавшись определениями (17), (20) и выполнив усреднение уравнений (13) и (14) по поверхности Ферми, получаем

$$
\begin{aligned}
\left\langle\left\langle M_{m}(\vec{p}, \Omega)\right\rangle\right\rangle_{\mathrm{FS}}= & M_{m}(\Omega)= \\
= & -\frac{1}{\beta} \sum_{\vec{p}_{1}} \sum_{\Omega_{1}} g_{m m}^{2} D\left(\Omega, \Omega_{1}\right) G_{m}\left(\vec{p}_{1}, \Omega_{1}\right)\left[1+g_{m m}^{2} P_{\mathrm{V} m}\left(Q_{\mathrm{c}}, \Omega, \Omega_{1}\right)\right]- \\
& \quad-\frac{1}{\beta} \sum_{\vec{p}_{1}, \Omega_{1}} g_{m n}^{2} D\left(\Omega, \Omega_{1}\right) G_{n}\left(\vec{p}_{1} \Omega_{1}\right)
\end{aligned}
$$

(здесь следует полагать $n \neq m$ ),

$$
\begin{aligned}
& \Sigma_{1}(\Omega)=-\frac{1}{\beta} \sum_{\vec{p}_{1}} \sum_{\Omega_{1}} D\left(\Omega, \Omega_{1}\right) \widetilde{V}_{11}\left(\Omega, \Omega_{1}\right) F_{1}\left(\vec{p}_{1}, \Omega_{1}\right)-\frac{1}{\beta} \sum_{\vec{p}_{1}, \Omega_{1}} D\left(\Omega, \Omega_{1}\right) g_{12}^{2} F_{2}\left(\vec{p}_{1}, \Omega_{1}\right), \\
& \Sigma_{2}(\Omega)=-\frac{1}{\beta} \sum_{\vec{p}_{1}} \sum_{\Omega_{1}} D\left(\Omega, \Omega_{1}\right) g_{21}^{2} F_{2}\left(\vec{p}_{1}, \Omega_{1}\right)-\frac{1}{\beta} \sum_{\vec{p}_{1}} \sum_{\Omega_{1}} D\left(\Omega, \Omega_{1}\right) \widetilde{V}_{22}\left(\Omega, \Omega_{1}\right) F_{2}\left(\vec{p}_{1}, \Omega_{1}\right),
\end{aligned}
$$

где

$$
\widetilde{V}_{11}\left(\Omega, \Omega_{1}\right)=g_{11}^{2}+N_{1} g_{11}^{4}\left[2 P_{\mathrm{V}_{1}}\left(Q_{\mathrm{c}}, \Omega, \Omega_{1}\right)+P_{\mathrm{V}_{1}}^{\prime}\left(Q_{\mathrm{c}}, \Omega, \Omega_{1}\right)+P_{C_{1}}\left(Q_{\mathrm{c}}, \Omega, \Omega_{1}\right)\right],
$$


величина $\widetilde{V}_{22}$ получается заменой $1 \rightarrow 2$ в формуле $(23) ;$ функции $P_{\mathrm{V}_{n}, \mathrm{C}_{n}}\left(Q_{\mathrm{c}}, \Omega, \Omega_{1}\right)$ соответствуют усредненным по поверхности Ферми функциям $(20) ; Q_{\mathrm{c}}=q_{\mathrm{c}} /\left(2 p_{\mathrm{F}}\right)$. Как следует из формул (21)-(23), вершинные и "пересекающиеся" функции дают вклады в константы внутризонного электрон-фононного взаимодействия $g_{11}^{2}$ и $g_{22}^{2}$, не затрагивая межзонное взаимодействие $g_{12}^{2}$.

В дальнейших расчетах воспользуемся простым законом дисперсии энергии электронов для $n$-й зоны

$$
\varepsilon_{n}(\vec{p})=\zeta_{n}+\frac{p^{2}}{2 m_{n}}-\mu .
$$

Перейдем в функциях (20) от суммирования по $\vec{p}_{2}$ и $\Omega_{2}$ к интегрированию по энергии и частоте стандартным образом (как это делается при $T=0)$ :

$$
\frac{1}{\beta} \sum_{\vec{p}_{2}} \sum_{\Omega_{2}} \Phi_{n}\left(\vec{p}_{2}, \Omega_{2}\right)=N_{n} \int_{0}^{2 \pi} \frac{d \varphi}{4 \pi} \int_{0}^{\pi} \sin \theta d \theta \int_{-\mu_{n}}^{W_{n}-\mu_{n}} d \varepsilon\left(\vec{p}_{2}\right) \frac{1}{2 \pi} \int_{-\infty}^{\infty} d \Omega_{2} \Phi_{n}\left(\vec{p}_{2}, \Omega_{2}\right),
$$

где

$$
N_{n}=\frac{m_{n} \vec{p}_{\mathrm{F}_{n}}}{2 \pi^{2}}, \quad \mu_{n}=\mu-\zeta_{n}
$$

Интегрирование по $\Omega_{2}$ в бесконечных пределах обусловленно приближением слабой связи $\left(\omega_{0} / T_{\mathrm{c}} \gg 1\right)$. Интегрирование по угловым переменным выполняется в соответствии с малостью передаваемого импульса $q\left(q \ll 2 p_{\mathrm{F}}\right)$. При этом применяется методика расчета, развитая для этого случая в работах [10], [13], [14].

Отсюда получаем для трехмерной системы при $Q_{\mathrm{c}}=q_{\mathrm{c}} /\left(2 p_{\mathrm{F}}\right) \ll \omega_{0} / \mu_{n}$ выражения

$$
\begin{aligned}
P_{\mathrm{V} n}\left(Q_{\mathrm{c}}, \Omega, \Omega_{1}\right)= & \frac{\omega_{0} A_{n}\left(\Omega, \Omega_{1}\right)}{\left(\Omega-\Omega_{1}\right)^{2}}-\frac{\omega_{0} E^{2}}{\left(\Omega-\Omega_{1}\right)^{4}}\left[A_{n}\left(\Omega, \Omega_{1}\right)-\left(\Omega-\Omega_{1}\right)^{2} B_{n}\left(\Omega, \Omega_{1}\right)\right] \frac{1}{2} Q_{\mathrm{c}}^{4}, \\
P_{\mathrm{C} n}\left(Q_{\mathrm{c}}, \Omega, \Omega_{1}\right)= & \frac{\omega_{0} A_{n}\left(\Omega,-\Omega_{1}\right)}{\left(\Omega+\Omega_{1}\right)^{2}}-\frac{\omega_{0} E^{2}}{\left(\Omega+\Omega_{1}\right)^{4}}\left[A_{n}\left(\Omega,-\Omega_{1}\right)-\left(\Omega+\Omega_{1}\right)^{2} B_{n}\left(\Omega,-\Omega_{1}\right)\right] \times \\
& \times \frac{22}{3} Q_{\mathrm{c}}^{4}+\frac{\omega_{0} E}{\left(\Omega+\Omega_{1}\right)^{2}} C_{n}\left(\Omega,-\Omega_{1}\right) Q_{\mathrm{c}}^{2},
\end{aligned}
$$

где $E=2 \varepsilon_{\mathrm{F}}$,

$$
\begin{aligned}
A_{n}\left(\Omega, \Omega_{1}\right)= & \frac{\Omega-\Omega_{1}}{2}\left[2 \operatorname{arctg} \frac{\Omega}{\omega_{0}}-\operatorname{arctg} \frac{\Omega}{W_{n}-\mu_{n}+\omega_{0}}-\operatorname{arctg} \frac{\Omega}{\mu_{n}+\omega_{0}}+\right. \\
& \left.+\operatorname{arctg} \frac{\Omega_{1}}{\mu_{n}+\omega_{0}}-2 \operatorname{arctg} \frac{\Omega_{1}}{\omega_{0}}+\operatorname{arctg} \frac{\Omega_{1}}{W_{n}-\mu_{n}+\omega_{0}}\right], \\
B_{n}\left(\Omega, \Omega_{1}\right)=- & \frac{\mu_{n}+\omega_{0}}{2\left[\left(\mu_{n}+\omega_{0}\right)^{2}+\Omega_{1}^{2}\right]^{2}}\left[\left(\mu_{n}+\omega_{0}\right)^{2}+2 \Omega_{1}^{2}-\Omega_{1}\right]- \\
& -\frac{W_{n}-\mu_{n}+\omega_{0}}{2\left[\left(W_{n}-\mu_{n}+\omega_{0}\right)^{2}+\Omega_{1}^{2}\right]^{2}}\left[\left(W_{n}-\mu_{n}+\omega_{0}\right)^{2}+2 \Omega_{1}^{2}-\Omega_{1}\right], \\
C_{n}\left(\Omega, \Omega_{1}\right)=\frac{1}{4} \ln \frac{\left(W_{n}-\mu_{n}+\omega_{0}\right)^{2}+\Omega^{2}}{\left(\mu_{n}+\omega_{0}\right)^{2}+\Omega^{2}}-\frac{1}{4} \ln \frac{\left(W_{n}-\mu_{n}+\omega_{0}\right)^{2}+\Omega_{1}^{2}}{\left(\mu_{n}+\omega_{0}\right)^{2}+\Omega_{1}^{2}}+ & +\Omega_{1}\left(\Omega-\Omega_{1}\right) \frac{1}{2}\left[\frac{1}{\left(\mu_{n}+\omega_{0}\right)^{2}+\Omega_{1}^{2}}-\frac{1}{\left(W_{n}-\mu_{n}+\omega_{0}\right)^{2}+\Omega_{1}^{2}}\right] .
\end{aligned}
$$


Как следует из этих формул, значения вершинных функций существенно зависят от заполнения энергетических зон. При серединном заполнении $\left(\mu_{n}=W_{n} / 2\right)$ эти выражения значительно упрощаются, в частности, коэффициент $C_{n}\left(\Omega, \Omega_{1}\right)$ обращается в нуль.

\section{4. КРИТИЧЕСКАЯ ТЕМПЕРАТУРА СВЕРХПРОВОДЯЩЕГО ПЕРЕХОДА $T_{\mathrm{c}}$ И КОЭФФИЦИЕНТ ИЗОТОПИЧЕСКОГО ЭФФЕКТА $\alpha$}

Как и при рассмотрении однозонной неадиабатической системы, положим в формулах (26), (27) $\Omega=0$ и $\Omega_{1}=\omega_{0}$ [10], [13] и введем перенормировку констант электрон-фононного взаимодействия в соответствии с этим предположением:

$$
\begin{gathered}
\tilde{\lambda}_{n n}=N_{n} \widetilde{V}_{n n}\left(0, \omega_{0}\right)=\lambda_{n n}+\lambda_{n n}^{2}\left[2 P_{\mathrm{V}_{n}}\left(Q_{\mathrm{c}}, \omega_{0}\right)+P_{\mathrm{C}_{n}}\left(Q_{\mathrm{c}}, \omega_{0}\right)\right], \\
\tilde{\lambda}_{n n}^{Z}=\lambda_{n n}\left[n+\lambda_{n n} P_{\mathrm{V} n}\left(Q_{\mathrm{c}}, \omega_{0}\right)\right], \quad n=1,2, \\
\lambda_{12}=V_{12} N_{2},
\end{gathered}
$$

где верхний индекс $Z$ указывает на происхождение перенормировки констант электрон-фононного взаимодействия (вследствие учета диагонального массового оператора $M_{n}$, определяющего также величины $Z_{n}$ ). В результате выражения для массовых операторов $M_{m}(\Omega)$ и система уравнений для $\Sigma_{m}(\Omega)$ приобретают вид

$$
\begin{gathered}
M_{m}(\Omega)=-\tilde{\lambda}_{m m}^{Z} \frac{1}{\beta N_{m}} \sum_{\vec{p}_{1}} \sum_{\Omega_{1}} D\left(\Omega, \Omega_{1}\right) G_{m}\left(\vec{p}_{1}, \Omega_{1}\right)- \\
-\frac{\lambda_{m n}}{\beta N_{n}} \sum_{\vec{p}_{1}} \sum_{\Omega_{1}} D\left(\Omega, \Omega_{1}\right) G_{n}\left(\vec{p}_{1}, \Omega_{1}\right), \quad m \neq n, \\
\Sigma_{1}(\Omega)=-\frac{\tilde{\lambda}_{11}}{\beta N_{1}} \sum_{\vec{p}_{1}} \sum_{\Omega_{1}} D\left(\Omega, \Omega_{1}\right) F_{1}\left(\vec{p}_{1}, \Omega_{1}\right)-\frac{\lambda_{12}}{\beta N_{2}} \sum_{\vec{p}_{1}, \Omega_{1}} D\left(\Omega, \Omega_{1}\right) F_{2}\left(\vec{p}_{1}, \Omega_{1}\right), \\
\Sigma_{2}(\Omega)=-\frac{\tilde{\lambda}_{22}}{\beta N_{2}} \sum_{\vec{p}_{1}} \sum_{\Omega_{1}} D\left(\Omega, \Omega_{1}\right) F_{2}\left(\vec{p}_{1}, \Omega_{1}\right)-\frac{\lambda_{21}}{\beta N_{1}} \sum_{\vec{p}_{1}} \sum_{\Omega_{1}} D\left(\Omega, \Omega_{1}\right) F_{1}\left(\vec{p}_{1}, \Omega_{1}\right) .
\end{gathered}
$$

Положим $\Sigma_{n}(\Omega)=Z_{n}(\Omega) \Delta_{n}(\Omega)$. Подставляя в $(30)$ выражения для $F,(\vec{p}, \Omega)$ из $(11)$ в линейном по $\Delta_{n}$ приближении $\left(T \sim T_{\mathrm{c}}\right)$ и выполняя интегрирование по энергии в соответствии с (25), получаем

$$
\begin{aligned}
\Delta_{1}(\Omega)= & \frac{\tilde{\lambda}_{11}}{Z_{1}} \frac{1}{\beta} \sum_{\Omega_{1}} \frac{\omega_{0}^{2}}{\left(\Omega-\Omega_{1}\right)^{2}+\omega_{0}^{2}} \frac{\Delta_{1}\left(\Omega_{1}\right)}{\left|\Omega_{1}\right|} \varphi_{1}\left(\Omega_{1}\right)+ \\
& +\frac{\lambda_{12}}{Z_{1}} \frac{1}{\beta} \sum_{\Omega_{1}} \frac{\omega_{0}^{2}}{\left(\Omega-\Omega_{1}\right)^{2}+\omega_{0}^{2}} \frac{\Delta_{2}\left(\Omega_{1}\right)}{\left|\Omega_{1}\right|} \varphi_{2}\left(\Omega_{1}\right), \\
\Delta_{2}(\Omega)= & \frac{\tilde{\lambda}_{22}}{Z_{2}} \frac{1}{\beta} \sum_{\Omega_{1}} \frac{\omega_{0}^{2}}{\left(\Omega-\Omega_{1}\right)^{2}+\omega_{0}^{2}} \frac{\Delta_{2}\left(\Omega_{1}\right)}{\left|\Omega_{1}\right|} \varphi_{2}\left(\Omega_{1}\right)+ \\
& +\frac{\lambda_{21}}{Z_{2} \beta} \sum_{\Omega_{1}} \frac{\omega_{0}^{2}}{\left(\Omega-\Omega_{1}\right)^{2}+\omega_{0}^{2}} \frac{\Delta_{1}\left(\Omega_{1}\right)}{\left|\Omega_{1}\right|} \varphi_{1}\left(\Omega_{1}\right),
\end{aligned}
$$


где

$$
\begin{gathered}
\varphi_{n}\left(\Omega_{1}\right)=\operatorname{arctg} \frac{W_{n}-\tilde{\mu}_{n}}{Z_{n}\left|\Omega_{1}\right|}+\operatorname{arctg} \frac{\tilde{\mu}_{n}}{Z_{n}\left|\Omega_{1}\right|}, \\
Z_{n}=Z_{n}(0)=1-\lim _{\Omega \rightarrow 0} \frac{1}{\Omega} \operatorname{Im} M_{n}(\Omega)=1+\lambda_{n n}^{Z} \frac{1}{2}\left[\frac{W_{n}-\mu_{n}}{W_{n}-\mu_{n}+\omega_{0}}+\frac{\mu_{n}}{\mu_{n}+\omega_{0}}\right]+ \\
+\lambda_{n m} \frac{1}{2}\left[\frac{W_{m}-\mu_{m}}{W_{m}-\mu_{m}+\omega_{0}}+\frac{\mu_{m}}{\mu_{m}+\omega_{0}}\right], \\
\tilde{\mu}_{n}=\mu_{n}-\operatorname{Re} M_{n}(0), \quad m \neq n .
\end{gathered}
$$

При исследовании системы уравнений (31) применим приближение, используемое в теории сверхпроводимости с учетом эффекта запаздывания [23],

$$
\frac{\omega_{0}^{2}}{\left(\Omega-\Omega_{1}\right)^{2}+\omega_{0}^{2}} \approx \frac{\omega_{0}^{2}}{\Omega^{2}+\omega_{0}^{2}} \frac{\omega_{0}^{2}}{\Omega_{1}^{2}+\omega_{0}^{2}},
$$

и введем обозначения

$$
\Delta_{n}(\Omega)=\frac{\omega_{0}^{2}}{\Omega^{2}+\omega_{0}^{2}} \Delta_{n}, \quad \Phi_{n}\left(T_{\mathrm{c}}\right)=T_{\mathrm{c}} \sum_{\Omega_{1}} \frac{\omega_{0}^{4}}{\left(\Omega_{1}^{2}+\omega_{0}^{2}\right)^{2}} \frac{1}{\left|\Omega_{1}\right|} \varphi_{n}\left(\Omega_{1}\right) .
$$

Подставляя (33), (34) в систему уравнений (31), приведем эту систему к виду

$$
\Delta_{1}=\frac{\tilde{\lambda}_{11}}{Z_{1}} \Delta_{1} \Phi_{1}\left(T_{\mathrm{c}}\right)+\frac{\lambda_{12}}{Z_{1}} \Delta_{2} \Phi_{2}\left(T_{\mathrm{c}}\right), \quad \Delta_{2}=\frac{\lambda_{21}}{Z_{2}} \Delta_{1} \Phi_{1}\left(T_{\mathrm{c}}\right)+\frac{\tilde{\lambda}_{22}}{Z_{2}} \Delta_{2} \Phi_{2}\left(T_{\mathrm{c}}\right) .
$$

Температура сверхпроводящего перехода $T_{\mathrm{c}}$ определяется из условия разрешимости системы уравнений (35). Полагая определитель этой системы уравнений равным нулю, получаем

$$
\left(\bar{\lambda}_{11} \bar{\lambda}_{22}-\bar{\lambda}_{12} \bar{\lambda}_{21}\right) \Phi_{1}\left(T_{\mathrm{c}}\right) \Phi_{2}\left(T_{\mathrm{c}}\right)-\bar{\lambda}_{11} \Phi_{1}\left(T_{\mathrm{c}}\right)-\bar{\lambda}_{22} \Phi_{2}\left(T_{\mathrm{c}}\right)+1=0,
$$

где $\bar{\lambda}_{11}=\tilde{\lambda}_{11} / Z_{1} ; \quad \bar{\lambda}_{22}=\tilde{\lambda}_{22} / Z_{2} ; \quad \bar{\lambda}_{12}=\lambda_{12} / Z_{1} ; \quad \bar{\lambda}_{21}=\lambda_{21} / Z_{2}$.

Функцию $\Phi_{n}\left(T_{\mathrm{c}}\right)$ нетрудно привести к виду

$$
\begin{aligned}
\Phi_{n}\left(T_{\mathrm{c}}\right)=\pi & T_{\mathrm{c}} \sum_{\Omega_{1}} \frac{\omega_{0}^{2}}{\Omega_{1}^{2}+\omega_{0}^{2}} \frac{1}{\left|\Omega_{1}\right|}-T_{\mathrm{c}} \sum_{\Omega_{1}} \frac{\omega_{0}^{2}}{\Omega_{1}^{2}+\omega_{0}^{2}} \frac{1}{\left|\Omega_{1}\right|}\left[\operatorname{arctg} \frac{\left|\Omega_{1}\right|}{\bar{W}_{n}-\bar{\mu}_{n}}+\right. \\
& \left.+\operatorname{arctg} \frac{\left|\Omega_{1}\right|}{\bar{\mu}_{n}}\right]-T_{\mathrm{c}} \sum_{\Omega_{1}} \frac{\omega_{0}^{2}\left|\Omega_{1}\right|}{\left(\Omega_{1}^{2}+\omega_{0}^{2}\right)^{2}} \varphi_{n}\left(\Omega_{1}\right) .
\end{aligned}
$$

Первый член этого выражения содержит логарифмическую особенность по величине

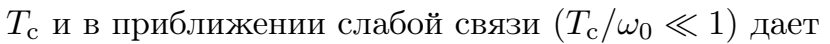

$$
\pi T_{\mathrm{c}} \sum_{\Omega_{1}} \frac{\omega_{0}^{2}}{\Omega_{1}^{2}+\omega_{0}^{2}} \frac{1}{\left|\Omega_{1}\right|} \approx \ln \frac{2 \omega_{0} \gamma}{\pi T_{\mathrm{c}}} .
$$

В том же приближении во всех остальных членах можно выполнить интегрирование по частоте в бесконечных пределах, как в случае $T=0$ (см. формулу (25)). Отсюда 
для функций $\Phi_{n}\left(T_{\mathrm{c}}\right)$ получаем

$$
\begin{gathered}
\Phi_{n}\left(T_{\mathrm{c}}\right)=\xi_{\mathrm{c}}+f_{n}, \quad \xi_{\mathrm{c}}=\ln \frac{2 \omega_{0} \gamma}{\pi T_{\mathrm{c}}}, \\
f_{n}=-\frac{1}{2}\left[\ln \left(1+m_{n}\right)+\ln \left(1+m_{n}^{\prime}\right)-\frac{1}{2}+\frac{1}{4}\left(\frac{m_{n}}{1+m_{n}}+\frac{m_{n}^{\prime}}{1+m_{n}^{\prime}}\right)\right], \\
m_{n}=\frac{\omega_{0}}{\bar{W}_{n}-\bar{\mu}_{n}}, \quad m_{n}^{\prime}=\frac{\omega_{0}}{\bar{\mu}_{n}}, \quad \bar{W}_{n}=\frac{W_{n}}{Z_{n}}, \quad \bar{\mu}_{n}=\frac{\mu_{n}}{Z_{n}} .
\end{gathered}
$$

Подставив (39) в уравнение (36), приведем последнее к виду

$$
\bar{a} \xi_{\mathrm{c}}^{2}-\bar{b} \xi_{\mathrm{c}}+\bar{c}=0
$$

где

$$
\begin{gathered}
\bar{a}=\bar{\lambda}_{11} \bar{\lambda}_{22}-\bar{\lambda}_{12} \bar{\lambda}_{21}, \quad \bar{b}=\bar{\lambda}_{11}+\bar{\lambda}_{22}-\bar{a}\left(f_{1}+f_{2}\right), \\
\bar{c}=1-\bar{\lambda}_{11} f_{1}-\bar{\lambda}_{22} f_{2}+\bar{a} f_{1} f_{2} .
\end{gathered}
$$

На основании (39) и (41) имеем

$$
T_{\mathrm{c}}=\frac{2 \omega_{0} \gamma}{\pi} e^{-\xi_{\mathrm{c}}}, \quad \xi_{\mathrm{c}}=\frac{\bar{b} \pm \sqrt{\bar{b}^{2}-4 \bar{a} \bar{c}}}{2 \bar{a}} .
$$

Это выражение формально совпадает со случаем обычного двухзонного сверхпроводника [2], [24]. Однако по существу оно значительно отличается, поскольку входящие в него величины перенормированы и содержат дополнительные вклады, определяемые неадиабатичностью системы Для перехода к однозонному случаю достаточно в приведенных выше формулах положить $N_{2}=0$. На этом пути получаем для критической температуры $T_{\mathrm{c}_{0}}=\left.T_{\mathrm{c}}\right|_{N_{2}=0}[13]$ соотношение

$T_{\mathrm{c}_{0}}=\frac{2 \omega_{0} \gamma}{\pi \sqrt{e}} \frac{\left[\left(\bar{W}_{1}-\bar{\mu}_{1}\right) \bar{\mu}_{1}\right]^{1 / 2}}{\left[\left(\bar{W}_{1}-\bar{\mu}_{1}+\omega_{0}\right)\left(\bar{\mu}_{1}+\omega_{0}\right)\right]^{1 / 2}} \exp \left\{-\frac{1}{\bar{\lambda}_{11}}+\frac{1}{4}\left(\frac{1}{\bar{W}_{1}-\bar{\mu}_{1}+\omega_{0}}+\frac{1}{\bar{\mu}_{1}+\omega_{0}}\right)\right\}$.

При серединном заполнении энергетической зоны $\mu_{1}=W_{1} / 2$ это выражение переходит в выражение, полученное в работе [10],

$$
T_{\mathrm{c}_{0}}^{0}=\frac{2 \omega_{0} \gamma}{\pi \sqrt{e}(1+m)} \exp \left\{-\frac{1}{\bar{\lambda}_{11}}+\frac{1}{2} \frac{m}{m+1}\right\}
$$

где $m=2 \omega_{0} / W_{1}$.

Таким образом, формула (43) демонстрирует влияние перекрытия двух энергетических полос на поверхности Ферми, а также неадиабатичности и сильных электронных корреляций на критическую температуру сверхпроводящего перехода при произвольном заполнении энергетических зон.

На основании формулы (43) выражение для коэффициента изотопического эффекта $\alpha$ можно привести к виду

$$
\alpha=-\frac{d \ln T_{\mathrm{c}}}{d \ln M}=\frac{1}{2}\left[1+\frac{d \ln \left(T_{\mathrm{c}} / \omega_{0}\right)}{d \ln \omega_{0}}\right]=\frac{1}{2}\left[1-\frac{d \xi_{\mathrm{c}}}{d \ln \omega_{0}}\right],
$$


где

$$
\begin{aligned}
\frac{d \xi_{\mathrm{c}}}{d \ln \omega_{0}}=- & \frac{\bar{b} \pm \sqrt{\bar{b}^{2}-4 \bar{a} \bar{c}}}{\bar{a}^{2}} \frac{d \bar{a}}{d \ln \omega_{0}}+\frac{1}{2 \bar{a}} \times \\
& \times\left\{\frac{d \bar{b}}{d \ln \omega_{0}} \pm \frac{1}{\sqrt{\bar{b}^{2}-4 \bar{a} \bar{c}}}\left[\frac{d \bar{b}}{d \ln \omega_{0}}-2 \bar{a} \frac{d \bar{c}}{d \ln \omega_{0}}-2 \bar{c} \frac{d \bar{a}}{d \ln \omega_{0}}\right]\right\}
\end{aligned}
$$

Как следует из (46) и (47), коэффициент $\alpha$ отличается от величины $1 / 2$, характерной для случая обычного сверхпроводника, зависимостью величин $\bar{a}, \bar{b}, \bar{c}$ от $\omega_{0}$. Эта зависимость возникает вследствие неадиабатичности системы и эффектов запаздывания в системе с электрон-фононным взаимодействием.

Отметим, что знак в формулах (43) и (47) выбирается, как и в случае адиабатических систем, из условий $\xi_{c}>0$ и максимальности величины $T_{\mathrm{c}}[25]$.

\section{5. ЧИСЛЕННЫЕ РАСЧЕТЫ И ОБСУЖДЕНИЕ РЕЗУЛЬТАТОВ}

В данной работе построена теория сверхпроводимости для двухзонной неадиабатической системы путем выхода за рамки теоремы Мигдала [6] в линейном по неадиабатичности приближении. Это означает, что в определении массовых операторов (диагональных $M_{n}$ и недиагональных $\Sigma_{n}, n=1,2$ ) учитываются дополнительные по сравнению с теорией Боголюбова-Элиашберга-Мигдала диаграммы с пересечением двух линий электрон-фононного взаимодействия, отвечающие вершинным функциям $P_{\mathrm{V} n}$ и "пересекающимся" функциям $P_{\mathrm{C} n}$. Используемое в работе приближение слабой связи $\left(T_{\mathrm{c}} / \omega_{0} \ll 1, \Delta_{n} / \omega_{0} \ll 1\right)$ позволяет получить аналитические выражения для этих функций и показать, что их поведение определяется значением передаваемого импульса $q$ при электрон-фононном взаимодействии. При малых значениях импульса обрезания электрон-фононного взаимодействия $q_{\mathrm{c}} \ll 2 p_{\mathrm{F}}$ функции $P_{\mathrm{Vn}}$ и $P_{\mathrm{C} n}$ дают положительный вклад, а при $q \sim 2 p_{\mathrm{F}}-$ отрицательный. Малость вели-

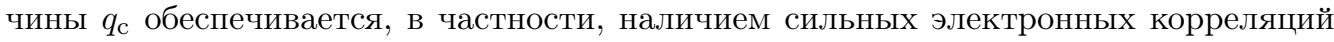
в системе [8] или квазиодномерным законом дисперсии энергии электронов [11].

При выполнении численных расчетов на основании формул (43) и (47) мы ограничились упрощенным случаем $m=m_{1}=m_{2} \quad\left(m_{n}=2 \omega_{0} / W_{n}\right)$ и серединным заполнением энергетических зон $\left(\mu_{n}=W_{n} / 2\right)$.

Пусть параметры теории суть $\lambda_{11}=0.5 ; \quad \lambda_{22}=0.3 ; \quad \lambda_{12}=0.1 ; \quad \lambda_{21}=0.05$. В соответствии с формулами (36), (28) и (32) эти параметры перенормируются вследствие эффектов неадиабатичности и сильных электронных корреляций. Значения перенормированных параметров $\bar{\lambda}_{n m}$ при различных значениях параметра Мигдала $m$ и при значениях $Q_{\mathrm{c}}=0.1$ и $Q_{\mathrm{c}}=0.9$, а также значения функций $P_{\mathrm{V}_{1}}$ и $P_{\mathrm{C}_{1}}$ приведены в таблице. 


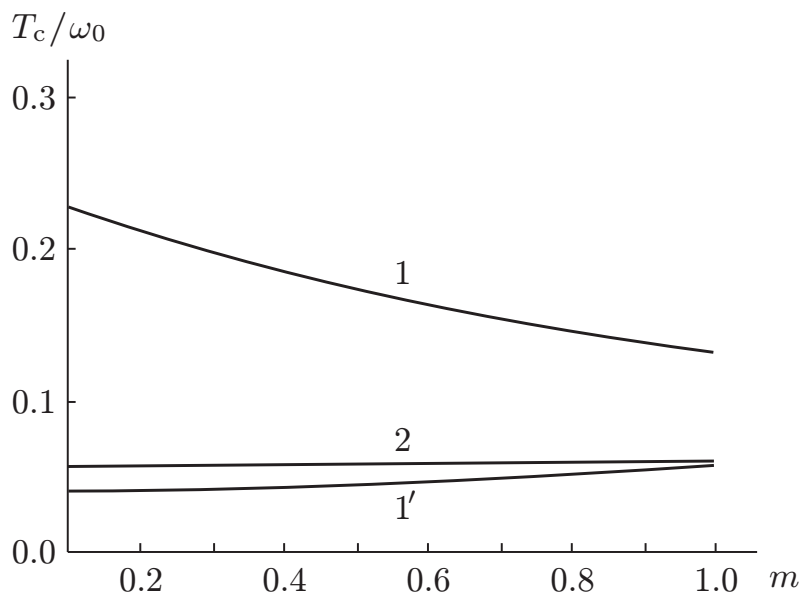

Рис. 1. Зависимость температуры сверхпроводящего перехода $T_{\mathrm{c}}$ от параметра Мигдала $m$. Кривые 1 и $1^{\prime}$ отвечают случаям неадиабатической двухзонной системы при $Q_{\mathrm{c}}=0.1$ и $Q_{\mathrm{c}}=0.9$, соответственно; кривая $2-$ случаю адиабатической системы.

\begin{tabular}{|c|c|c|c|c|c|c|c|}
\hline & $\bar{m}$ & $P_{\mathrm{V}_{1}}$ & $P_{\mathrm{C}_{1}}$ & $\bar{\lambda}_{11}$ & $\bar{\lambda}_{22}$ & $\bar{\lambda}_{12}$ & $\bar{\lambda}_{21}$ \\
\hline \multirow{5}{*}{$Q_{\mathrm{c}}=0.1$} & 0 & 0 & 0 & 0.312 & 0.24 & 0.065 & 0.038 \\
& 0.1 & 0.679 & 0.656 & 0.59 & 0.35 & 0.058 & 0.036 \\
& 0.3 & 0.565 & 0.553 & 0.584 & 0.344 & 0.064 & 0.038 \\
& 0.6 & 0.426 & 0.425 & 0.568 & 0.333 & 0.069 & 0.04 \\
& 0.9 & 0.342 & 0.342 & 0.556 & 0.32 & 0.073 & 0.042 \\
\hline \multirow{5}{*}{$Q_{\mathrm{c}}=0.9$} & 0 & 0 & 0 & 0.312 & 0.24 & 0.065 & 0.038 \\
& 0.1 & -0.053 & -0.044 & 0.315 & 0.223 & 0.065 & 0.038 \\
& 0.3 & -0.012 & -0.096 & 0.322 & 0.227 & 0.068 & 0.039 \\
& 0.6 & -0.008 & -0.112 & 0.339 & 0.236 & 0.073 & 0.041 \\
& 0.9 & 0.005 & -0.113 & 0.36 & 0.245 & 0.076 & 0.042 \\
\hline
\end{tabular}

Видно, что поведение перенормированных констант электрон-фононного взаимодействия не однозначно и определяется как значением импульса обрезания электронфононного взаимодействия $Q_{\mathrm{c}}$, так и значением параметра Мигдала $m$. Отношение $T_{\mathrm{c}} / \omega_{0}$ как функция от $m$ приведено на рис. 1 . При вычислении этого отношения мы исходили из формулы (43), полученные численные результаты умножили на $\sqrt{e}$. Такая поправка необходима, поскольку используемое в работе приближение (33) занижает величину $T_{\mathrm{c}}$ на множитель порядка $e^{-1 / 2}[26]$. На рис. 1 кривые $1,1^{\prime}$ отвечают случаям двухзонной неадиабатической системы при $Q_{\mathrm{c}}=0.1, Q_{\mathrm{c}}=0.9$, соответственно; кривая 2 - случаю двухзонной адиабатической системы. Сравнение этих кривых показывает, что эффекты неадиабатичности и сильные электронные корреляции существенно увеличивают температуру сверхпроводящего перехода $T_{\mathrm{c}}$

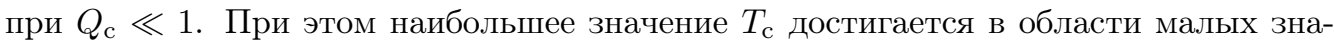
чений параметра $m(m \sim 0.1)$. При $Q_{\mathrm{c}} \sim 1$ имеем уменьшение величины $T_{\mathrm{c}}$ и ее слабую зависимость от параметра $m$. 


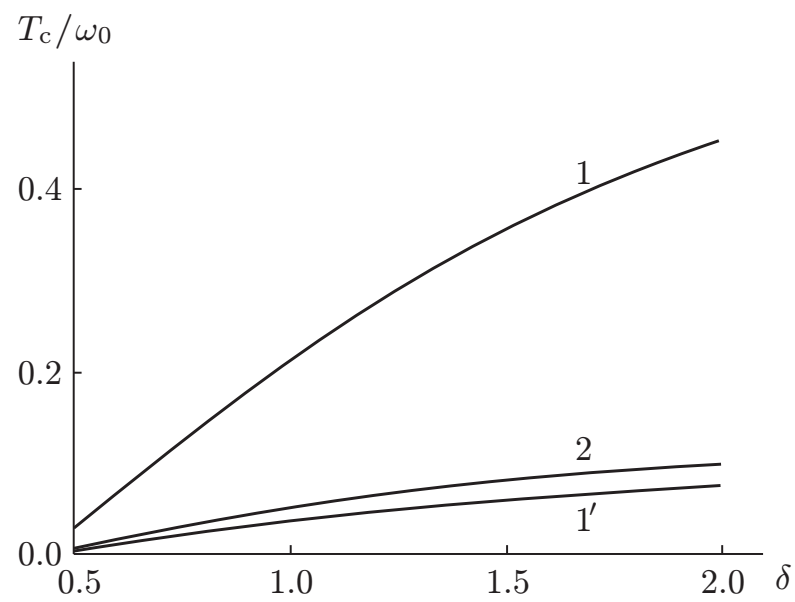

Рис. 2. Зависимость величины $T_{\text {c }}$ от коэффициента пропорционального увеличения значений констант электрон-фононного взаимодействия внутризонных $\lambda_{n n}$ и межзонных $\lambda_{n n_{1}}\left(n, n_{1}=1,2\right)$ при значении параметра Мигдала $m=0.2$. Обозначения кривых те же, что и на рис. 1.

Эти результаты говорят о том, что в системах с малыми значениями отношения энергии Дебая к энергии Ферми вклад вершинных поправок в сверхпроводимость может быть существенным, если передаваемый импульс мал.

$\mathrm{K}$ веществам с малыми значениями $m(m \sim 0.1)$ относится, в частности, двухзонный сверхпроводник $\mathrm{Mg} \mathrm{B}_{2}$. Наличие сильных электронных корреляций или особенностей в электронном энергетическом спектре в такого рода системах может способствовать значительному усилению влияния эффектов неадиабатичности на сверхпроводимость.

На рис. 2 приведена зависимость отношения $T_{\mathrm{c}} / \omega_{0}$ от коэффициента $\delta$, определяющего пропорциональное увеличение всех констант электрон-фононного взаимодействия в двухзонной модели $\left(\lambda_{n m}^{\prime}=\delta \lambda_{n m}\right)$. Рассмотрены случаи $m=0.2, Q_{\mathrm{c}}=0.1$ (кривая 1$)$ и $m=0.2, Q_{\mathrm{c}}=0,9$ (кривая $\left.1^{\prime}\right)$. Кривая 2 отвечает адиабатической си-

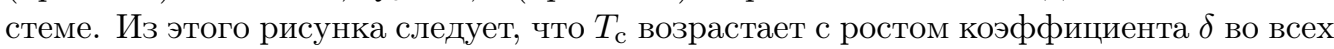
случаях.

На рис. 3 приведена зависимость коэффициента изотопического эффекта $\alpha$ от параметра Мигдала $m$. При $Q_{\text {c }} \ll 1$ и $m<0.1$ коэффициент $\alpha>0.5$, затем его величина значительно убывает с ростом $m$ и достигает значения $\alpha \approx 0.2$ (кривая 1 ). При $Q_{\mathrm{c}} \sim 1$ величина $\alpha$ слабо убывает, а затем растет с увеличением параметра $m$, оставаясь приблизительно равной 0.5 (кривая $1^{\prime}$ ).

Таким образом, эффекты неадиабатичности в двухзонных сверхпроводниках при малых значениях импульса обрезания электрон-фононного взаимодействия $Q_{\text {c }}$ даже при наличии промежуточной электрон-фононной связи позволяют достичь высоких значений температуры сверхпроводящего перехода $T_{\mathrm{c}}$ и малых значений изотопического эффекта. Такая картина наблюдается, например, в высокотемпературных оксидных металлах. 


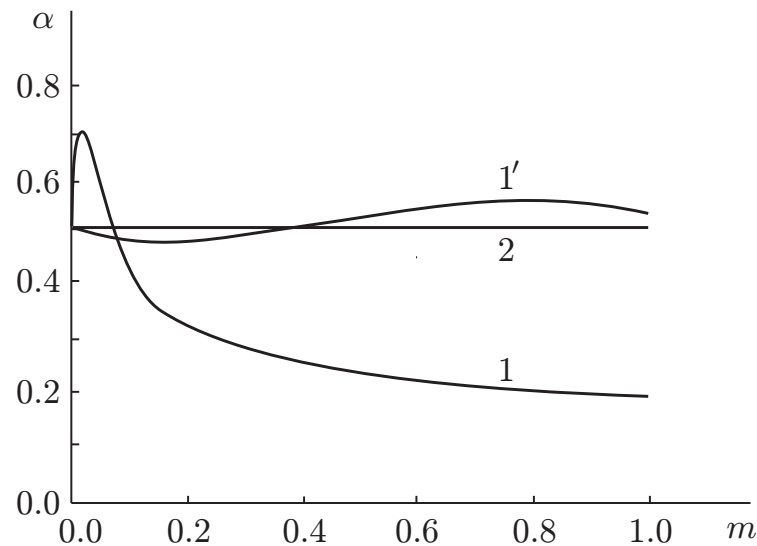

Рис. 3. Зависимость изотопического коэффициента $\alpha$ от параметра Мигдала. Обозначения кривых те же, что и на рис. 1

Приведенные выше численные результаты выполнены при $\lambda_{11}>\lambda_{22}>\lambda_{12}>\lambda_{21}$. Полученные результаты находятся в качественном согласии со случаем однозонного сверхпроводника [10].

В предельном случае $\lambda_{11}=\lambda_{22}=0, \lambda_{12}$ и $\lambda_{21} \neq 0$ рассмотренные эффекты неадиабатичности не влияют на сверхпроводимость, поскольку в этом предельном случае константы межзонного взаимодействия $\lambda_{n m}$ не подвергаются перенормировке из-за учета вершинных функций.

Отметим, что возможность возникновения высокотемпературной сверхпроводимости в системе с сильными электронными корреляциями на основе фононного механизма и сверхпроводимости обсуждалась во многих работах (см., например, [27]).

\section{Список литературы}

[1] J. G. Berdnorz, K. A. Müller, Z. Phys. B, 64 (1986), 189.

[2] В. А. Москаленко, Физика металлов и металловед., 8 (1959), 503.

[3] H. Suhl, B. T. Matthias, L. R. Walker, Phys. Rev. Lett., 3 (1959), 552.

[4] M. E. Palistrant, Moldav. J. Phys. Sciences, 3:2 (2004), 133; cond-mat/0305496; L.Z. Kon, Some kinetic properties of the two-band superconductors, cond-mat/0309707.

[5] В.А. Москаленко, М.Е. Палистрант, В. М. Вакалюк, УФН, 161 (1991), 155; cond-mat/0309671.

[6] А.Б. Мигдал, ЖЭТФ, 34 (1958), 1438.

[7] O. V. Danylenko, O. V. Dolgov, Nonadiabatic Contribution to the Quasiparticle Self-Energy in Systems with Strong Electron-Phonon Interaction, cond-mat/0007189.

[8] M. L. Kulić, R. Zeyher, Phys. Rev. B, 49 (1994), 4395; R. Zeyher, M. L. Kulić, Phys. Rev. B, 53 (1996), 2850.

[9] L. Pietronero, S. Strässler, C. Grimaldi, Phys. Rev. B, 52 (1995), 10516.

[10] C. Grimaldi, L. Pietronero, S. Strassler, Phys. Rev. B, 52 (1995), 10530.

[11] М.Е. Палистрант, Физика низк. темn, 31 (2005), 500.

[12] A. A. Abrikosov, Y.C. Campuzano, K. Gofron, Physica C, 214 (1993), 73.

[13] ; M. E. Palistrant, F. G. Kochorbe, J. Superconduct. Incorp. Novel Magnet., 15 (2002), 113; J. Phys. Condens. Matt., 15 (2003), 3267; Int. J. of Modern Phys. B, 17 (2003), 2545; М.Е. Палистрант, Физика низк. темп., 29 (2003), 1173. 
[14] М.Е. Палистрант, Физика низк. темn., 26 (2000), 557; 28 (2002), 157; ТМФ, 135 (2003), 137.

[15] J. Nagamatsu, N. Nakagawa, T. Muranaka et al., Nature (London), 463 (2001), 401.

[16] P. C. Canfield, S. L. Bud'ko, D. K. Finnemore, Physica C, 385 (2003), 1.

[17] F. Bouquet, Y. Wang, I. Sheikin et al, Physica C, 385 (2003), 192.

[18] T. Mishonov, S. L. Drechsler, E. Penev, Mod. Phys. Lett. B, 17 (2003), 755; cond-mat/0209192; T. Mishonov, E. Penev, J. O. Indekeu, V. I. Pokrovsky, Phys. Rev. B, 68 (2003), 104517; cond-mat/0209342; O. V. Dolgov, R. K. Kremer, J. Kortus et al., Phys. Rev. B, 72 (2005), 024504.

[19] A.S. Alexandrov, Breakdown of the Migdal-Eliashberg theory in the strong-coupling adiabatic regime, cond-mat/0102189.

[20] H. Krakauer, E. Pickett, Phys. Rev. Lett., 60 (1988), 1665; J. F. Herman, R. V. Kasowski, W. G. Hsu, Phys. Rev. B, 36 (1987), 6904; J. Kortus, I. I. Mazin, K. D. Belashchenko, V.P. Antropov, L. L. Boyer, Phys. Rev. Lett., 86 (2001), 4656; J. M. An, W. E. Pickett, Phys. Rev. Lett., 86 (2001), 4366.

[21] А. А. Абрикосов, Л. П. Горьков, И. Е. Дзялошинский, Методы квантовой теории поля в статистической физике, Физматгиз, М., 1962.

[22] Ф.Г. Кочорбэ, М.Е. Палистрант, ЖЭТФ, 104 (1993), 3084; ТМФ, 96 (1993), 459; M. E. Palistrant, Int. J. Modern Phys. B, 19 (2005), 929.

[23] W. L. Mc Millan, Phys. Rev., 167 (1968), 331.

[24] В. А. Москаленко, Л. З. Кон, М.Е. Палистрант, Низкотемпературные свойства металлов с особенностями зонного спектра, Штиинца, Кишинев, 1989.

[25] M. E. Palistrant, F. G. Kochorbe, Physica C, 194 (1992), 351.

[26] R. Combescot, Phys. Rev. B, 42 (1990), 7810.

[27] J. H. Kim, Z. Tešanović, Phys. Rev. Lett., 71 (1993), 4218; A. Lanzara, P. V. Bogdanov, X. J. Zhow et al., Nature, 412 (2001), 510; В. А. Москаленко, П. Ентил, М. Маринаро, Д.Ф. Дигор, ЖЭТФ, 124 (2003), 700.

Поступила в редакцию 26.02.2006, после доработки 31.03.2006 